\title{
Theoretical Analysis of the Noise Power Ratio of Nonlinear Power Amplifiers
}

\author{
Rajendra Kumar \\ Department of Electrical Engineering, California State University, Long Beach, CA 90840, USA \\ Correspondence should be addressed to Rajendra Kumar; rajendra.kumar@csulb.edu
}

Received 29 July 2016; Revised 27 October 2016; Accepted 31 October 2016

Academic Editor: Mehmet Sezer

Copyright (C) 2016 Rajendra Kumar. This is an open access article distributed under the Creative Commons Attribution License, which permits unrestricted use, distribution, and reproduction in any medium, provided the original work is properly cited.

\begin{abstract}
This paper presents a theoretical analysis and derives the amplifier output noise power spectral density result in a closed form when the input to the amplifier is a band limited Gaussian noise. From the computed power spectral density the NPR is evaluated by a simple subtraction. The method can be applied to any amplifier with known input-output characteristics. The method may be applied to analyze various other important characteristics of the nonlinear amplifier such as spectral regrowth that refers to the spreading of the signal bandwidth when a band limited signal is inputted to the nonlinear amplifier. The paper presents numerical results on the NPR as a function of the noise bandwidth, depth level of the notch, and the output power back-off obtained from the analysis presented in the paper.
\end{abstract}

\section{Introduction}

The power amplifier is one of the most important subsystems of modern communication systems [1-15]. In the case of satellite downlinks, the power efficiency of the high-power amplifiers (HPAs) is very important as the requirements on the power directly translate into the size, weight, and cost of the satellite payload. As the power efficiency is relatively high when the amplifier is operated near the saturation region, in the case of satellite links, the HPA by necessity needs to be operated in such a region. However, the nonlinearity of the amplifier in the near saturation region introduces considerable distortion in the signal to be amplified. As the output power back-off is reduced the signal to distortion power ratio at the amplifier output is correspondingly reduced. This places a serious restriction on the amount of backoff that needs to be introduced resulting in a loss of the available output power and equally importantly in a reduced power conversion efficiency thus resulting in an increased demand on the D.C. power which in case is provided by solar panels and thus has a direct implication on the size, weight, and cost of the satellite payload. In wireless communication systems any reduction in the power conversion efficiency results in a corresponding reduction in the battery life at the user terminal. It is thus extremely important to analyze the performance when the power amplifier is operated with relatively small output power back-off thereby exhibiting significant nonlinear behavior.

Among the various methods to assess the performance of an amplifier is the evaluation of the signal to distortion ratio at the amplifier output as a function of the amplifier output power back-off. In case of digitally modulated signals at the amplifier input in the digital communication systems, one of the most important performance measures is in terms of the bit error rate (BER) achieved in the presence of both the distortion introduced by the amplifier and any channel interference plus noise. However, both these measures are functions of the detailed modulation techniques, multiple accessing or multiplexing techniques, and the number of user signals at the amplifier input in addition to the amplifier input-output characteristics and the output power backoff. The various performance measures may be obtained by detailed mathematical analysis, computer simulations, and/or experimental measurements. Both the simulations and experimental measurements are, in general, very time consuming and expensive while the analysis methods are generally difficult and are for more specific cases. For example, detailed analysis has been presented by the author for 
the case of the code division multiple accessing (CDMA) [1-3] with an approximate analysis based on the Gaussian assumption on the nonlinear distortion appearing in earlier literature [4-6]. An infinite series result for the output of a nonlinear device in terms of the autocorrelation function of an input Gaussian process is presented in $[7,8]$. An analysis for the distortion effects of the nonlinear amplifier on OFDM signals appears in $[9,10]$. Extensive relatively earlier literature exists on the effects of the nonlinear amplifier on FDMA signals as in [13-17] and their references.

A performance characterization of the nonlinear power amplifiers that is independent of the specifics of the amplifier input signal, such as the multiple accessing and modulation techniques used, the number of users, and the relative power level of the various user signals, is commonly used in the practice. This measure is termed the noise power ratio (NPR). The noise power ratio is measured by inputting the amplifier with a white noise of bandwidth equal to the specified signal bandwidth. A notch in the input noise band is created with the bandwidth of the notch much smaller than the noise bandwidth. At the output of the amplifier one measures the noise power spectral density both inside and outside the notch with the ratio of the two by definition equal to the amplifier NPR that is a function of the total output noise power or the output power back-off.

While the NPR measurements are relatively less intensive compared to some other performance measurements such as the BER measurements, nevertheless these do require extensive measurements as well, in which from the measurements alone it is not possible to predict the performance for the amplifiers other than the one involved in the measurement; in other words it does not provide any measure of the sensitivity of the NPR to the amplifier input-output characteristics and does not address the validation issues of the experimental data. Thus it is of great interest to be able to evaluate the amplifier NPR by independent analytical means. This paper presents a theoretical analysis of the NPR for the nonlinear amplifier that is also applicable to any other nonlinear devices.

This paper presents a theoretical analysis and derives the amplifier output noise power spectral density result in a closed form when the input to the amplifier is a band limited noise with a notch in the spectral band. From the computed noise power spectral density (PSD) the NPR is evaluated as the ratio of the PSDs evaluated outside and inside of the notch. The method can be applied to any amplifier with known input-output characteristics. The paper presents numerical results on the NPR as a function of the noise bandwidth, depth level of the notch, and the output power back-off obtained from the analysis presented in the paper.

\section{Amplifier Model}

It is assumed in this analysis that the amplifier output $y(t)$ can be expressed in terms of its input bandpass process $u(t)$ via its input-output characteristic function $F(u)$ as

$$
y(t)=F(u(t))
$$

With a power series expansion of $F(u)$ about $u=0$, the amplifier output $y(t)$ may be expressed in the following series form:

$$
y(t)=K_{1} u(t)+K_{3} u^{3}(t)+K_{5} u^{5}(t)+\cdots,
$$

where the coefficients $K_{1}, K_{3}, \ldots$ may be obtained from the amplifier characteristics using a Taylor series expansion or by a finite degree polynomial approximation [1].

\section{Correlation Function of the Amplifier Output Process}

The correlation function of the amplifier output process denoted by $R_{y}(\tau)$ may be obtained from (2) as

$$
\begin{aligned}
& R_{y}(\tau)=E[y(t) y(t+\tau)] \\
& =E\left[\left\{K_{1} u(t)+K_{3} u^{3}(t)+\cdots\right\}\right. \\
& \left.\cdot\left\{K_{1} u(t+\tau)+K_{3} u^{3}(t+\tau)+\cdots\right\}\right],
\end{aligned}
$$

where $E$ denotes the expected value operator. Carrying out the multiplication of the two series in (3) and grouping the terms of the same order yield

$$
\begin{aligned}
& R_{y}(\tau)=K_{1}^{2} E[u(t) u(t+\tau)] \\
& +\left\{K_{1} K_{3} E\left[u(t) u^{3}(t+\tau)\right]\right. \\
& \left.+K_{1} K_{3} E\left[u^{3}(t) u(t+\tau)\right]\right\} \\
& +\left\{K_{1} K_{5} E\left[u(t) u^{5}(t+\tau)\right]\right. \\
& +K_{3}^{2} E\left[u^{3}(t) u^{3}(t+\tau)\right] \\
& \left.+K_{1} K_{5} E\left[u^{5}(t) u(t+\tau)\right]\right\}+\cdots .
\end{aligned}
$$

Denoting by $\xi(t)$ the normalized process $u(t) / \sigma$ with $\sigma^{2}$ denoting the variance of the process $u(t)$, the correlation function $R_{y}(\tau)$ may be expressed as

$$
\begin{aligned}
& R_{y}(\tau) \\
& =\sigma^{2} K_{1}^{2} E\left[\xi \xi_{\tau}\right]+\sigma^{4}\left\{K_{1} K_{3} E\left[\xi \xi_{\tau}^{3}\right]+K_{1} K_{3} E\left[\xi^{3} \xi_{\tau}\right]\right\} \\
& \quad+\sigma^{6}\left\{K_{1} K_{5} E\left[\xi \xi_{\tau}^{5}\right]+K_{3}^{2} E\left[\xi^{3} \xi_{\tau}^{3}\right]+K_{1} K_{5} E\left[\xi^{5} \xi_{\tau}\right]\right\} \\
& \quad+\cdots
\end{aligned}
$$

where $\xi$ and $\xi_{\tau}$ denote $\xi(t)$ and $\xi(t+\tau)$, respectively, for the convenience of notations. Assuming that $u(t)$ is a zero mean process, the moments $E\left[\xi^{n} \xi_{\tau}^{m}\right]$ for any pair of nonnegative integers may be obtained by the following integral:

$$
E\left[\xi^{n} \xi_{\tau}^{m}\right]=\int_{-\infty}^{\infty} \int_{-\infty}^{\infty} \xi_{1}^{n} \xi_{2}^{m} f\left(\xi_{1}, \xi_{2}\right) d \xi_{1} d \xi_{2} .
$$

In (6) the function $f\left(\xi_{1}, \xi_{2}\right)$ denotes the joint pdf (probability density function) of the random variables $\xi$ and $\xi_{\tau}$ or by 
definition the two-dimensional pdf of the process $\xi(t)$. For the case of Gaussian process assumed in this report, the joint pdf $f\left(\xi_{1}, \xi_{2}\right)$ is given by

$$
f\left(\xi_{1}, \xi_{2}\right)=\frac{1}{2 \pi \sqrt{1-\rho^{2}}} \exp \left\{-\frac{\xi_{1}^{2}-2 \rho \xi_{1} \xi_{2}+\xi_{2}^{2}}{1-\rho^{2}}\right\}
$$

where $\rho$ denotes the correlation coefficient between $\xi$ and $\xi_{\tau}$. In general $\rho$ is a function of $\tau$; however for notational convenience the argument $\tau$ of $\rho$ has been dropped in (7). Following the approach of $[18,19]$, the function $f\left(\xi_{1} \xi_{2}\right)$ is expressed in terms of the following series so as to evaluate the integral in (6) in closed form:

$$
f\left(\xi_{1}, \xi_{2}\right)=\sum_{p=0}^{\infty} g_{p}\left(\xi_{1}\right) g_{p}\left(\xi_{2}\right) \frac{\rho^{p}}{p !} .
$$

In (8) the functions $g_{p}(x)$ are given by

$$
\begin{aligned}
& g_{0}(x)=\frac{1}{\sqrt{2 \pi}} \exp \left(-\frac{x^{2}}{2}\right) ; \\
& g_{p}(x)=\frac{d^{p} g_{0}(x)}{d x^{p}} ; \quad p=1,2, \ldots
\end{aligned}
$$

In order to obtain closed form expressions for the desired moments, the functions $g_{p}(x)$ are related to the Hermite polynomials. For example,

$$
\begin{aligned}
& \frac{d^{n}}{d z^{n}}\left\{\exp \left(\frac{z^{2}}{2}\right)\right\}=C_{n}(z) \exp \left(\frac{z^{2}}{2}\right) ; \\
& n=0,1,2, \ldots, \\
& C_{n}(z)=z C_{n-1}(z)+(n-1) C_{n-2}(z) ; \\
& n=2,3, \ldots \text {, } \\
& C_{0}(z)=1 ; \\
& C_{1}(z)=z \text {. }
\end{aligned}
$$

In (10), $C_{n}(z)$ denotes the Hermite polynomial of degree $n$. Substituting $z=j x$ with $j=\sqrt{-1}$, one obtains

$$
\begin{aligned}
\frac{d^{n}}{d z^{n}\left\{\exp \left(-\frac{x^{2}}{2}\right)\right\}}=j^{n} C_{n}(j x) \exp \left(-\frac{x^{2}}{2}\right) ; \\
n=0,1,2, \ldots, \\
C_{n}(j x)=j x C_{n-1}(j x) \\
+(n-1) C_{n-2}(j x) ; \\
n=2,3, \ldots ; \\
C_{0}(j x)=1 ; \\
C_{1}(z)=j x .
\end{aligned}
$$

Multiplication on both sides of (12) by $j^{n} \exp \left(-x^{2} / 2\right) / \sqrt{2 \pi}$ and application of (11) result in the following desired recursive expression for the functions $g_{p}(x)$ that are the derivatives of the function $g_{0}(x)=(1 / \sqrt{2 \pi}) \exp \left(-x^{2} / 2\right)$;

$$
\begin{aligned}
& g_{p}(x)=-x g_{p-1}(x)-(p-1) g_{p-2}(x) ; \\
& p=1,2, \ldots ; \\
& g_{0}(x)=\exp \frac{-x^{2} / 2}{\sqrt{2 \pi}} .
\end{aligned}
$$

Now from (6) and (8), one obtains

$$
E\left[\xi^{n} \xi_{\tau}^{m}\right]=\sum_{i=0}^{\infty} \eta_{n, i} \eta_{m, i} \frac{\rho^{i}(\tau)}{i !}
$$

with

$$
\eta_{k, i}=\int_{-\infty}^{\infty} x^{k} g_{i}(x) d x ; \quad k, i=0,1,2, \ldots
$$

Multiplying both sides of (13) by $x^{k}$ and integration over the interval $(-\infty, \infty)$ and using (15) yield the following recursive expression for $\eta_{k, i}$ :

$$
\begin{aligned}
& \eta_{k, i}=-\eta_{k+1, i-1}-(i-1) \eta_{k, i-2} ; \quad i, k=1,2, \ldots, \\
& \eta_{0,0}=1 ; \\
& \eta_{0, i}=0 \quad \text { for } i>0 .
\end{aligned}
$$

The coefficients $\eta_{k, 0}$ which are the moments of the Gaussian distribution can be obtained by direct integration and are given by

$$
\eta_{k, 0}= \begin{cases}1 \cdot 3 \cdot 5 \cdots(k-1) ; & k \text { even; } k>0 \\ 0 ; & k \text { odd }\end{cases}
$$

With the initial conditions in (17) and (18), $\eta_{k, i}$ for $i, k>0$ may be computed from (16). For example, from (16) $\eta_{k, 1}=-\eta_{k+1,0}$ and in view of (17) one obtains

$$
\eta_{k, 1}= \begin{cases}-1 \cdot 3 \cdot 5 \cdots k ; & k \text { odd } ; k>0 \\ 0 ; & k \text { odd } .\end{cases}
$$

Similarly, from (16), (18), and (19)

$$
\begin{aligned}
\eta_{k, 2} & =-\eta_{k+1,1}-\eta_{k, 0} \\
\eta_{k, 2} & =\{1 \cdot 3 \cdot 5 \cdots(k+1)\}-\{1 \cdot 3 \cdot 5 \cdots(k-1)\} \\
& =\{1 \cdot 3 \cdot 5 \cdots(k-1)\}\{(k+1)-1\} \\
& =1 \cdot 3 \cdot 5 \cdots(k-1) \cdot k ; \quad k \text { even; } k>0, \\
\eta_{k, 2} & =0 ; \quad k \text { odd. }
\end{aligned}
$$

In general, $\eta_{k, i}$ have the following properties which follow from the recursion (15)-(17):

(i)

$$
\eta_{k, i}=0 \quad \text { for } i>k
$$


TABLE 1: Sample values of the coefficients $\eta_{k, i}$

\begin{tabular}{ccccccc}
\hline$k$ & & \multicolumn{5}{c}{$i$} \\
& 0 & 1 & 2 & 3 & 4 & 5 \\
\hline 0 & 1 & 0 & 0 & 0 & 0 & 0 \\
1 & 0 & -1 & 0 & 0 & 0 & 0 \\
2 & 1 & 0 & $2 \cdot 1$ & 0 & 0 & 0 \\
3 & 0 & $-3 \cdot 1$ & 0 & $-3 \cdot 2 \cdot 1$ & 0 & 0 \\
4 & $3 \cdot 1$ & 0 & $4 \cdot 3 \cdot 1$ & 0 & $4 \cdot 3 \cdot 2 \cdot 1$ & 0 \\
5 & 0 & $-5 \cdot 3 \cdot 1$ & 0 & $-5 \cdot 4 \cdot 3 \cdot 1$ & 0 & $-5 \cdot 4 \cdot 3 \cdot 2 \cdot 1$ \\
\hline
\end{tabular}

(ii)

For $i \leq k$ the only nonzero elements are those

for which $(k-i)$ is an even integer.

(iii) The nonzero elements $\eta_{k, i}$ are given by

$$
\begin{aligned}
\eta_{k, i}= & (-1)^{k}\{k \cdot(k-1) \cdots(k-i+1)\} \\
& \cdot\{(k-i-1) \cdot(k-i-3) \cdots 1\} .
\end{aligned}
$$

In (21a)-(21c) the first bracketed term has $i$ factors and is equal to 1 for $i=0$, with the second bracketed term having $(i-k) / 2$ factors. The value of $\eta_{k, i}$ can be computed using (i)(iii) for any integers $k, i$, and some of these terms are presented in Table 1.

In view of (21a)-(21c), the expression in (14) may be modified to that in (25)

$$
E\left[\xi^{n} \xi_{\tau}^{m}\right]=\sum_{i=0}^{\min (n, m)} \eta_{n, i} \eta_{m, i} \frac{\rho^{i}(\tau)}{i !} .
$$

Equation (22) also shows that the expression for $E\left[\xi^{n} \xi_{\tau}^{m}\right]$ is symmetric in $n$ and $m$ as expected from the stationarity of the process $\xi(t)$. For the case of $n=m$, with $n$ being odd, the expression for $E\left[\xi^{n} \xi_{\tau}^{m}\right]$ reduces to

$$
E\left[\xi^{n} \xi_{\tau}^{n}\right]=\sum_{\substack{i=1, i: \text { odd }}}^{n} \eta_{n, i}^{2} \frac{\rho^{i}(\tau)}{i !} .
$$

For example,

$$
\begin{aligned}
E\left[\xi^{3} \xi_{\tau}^{3}\right] & =\eta_{3,1}^{2} \rho(\tau)+\frac{\eta_{3,3}^{2} \rho^{3}(\tau)}{3 !}=9 \rho(\tau)+6 \rho^{3}(\tau) \\
E\left[\xi^{5} \xi_{\tau}^{5}\right] & =\eta_{5,1}^{2} \rho(\tau)+\frac{\eta_{5,3}^{2} \rho^{3}(\tau)}{3 !}+\frac{\eta_{5,5}^{2} \rho^{5}(\tau)}{5 !} \\
& =225 \rho(\tau)+600 \rho^{3}(\tau)+120 \rho^{5}(\tau)
\end{aligned}
$$

From (22)

$$
\begin{aligned}
& E\left[\xi^{3} \xi_{\tau}\right]=3 \rho(\tau) \\
& E\left[\xi^{5} \xi_{\tau}\right]=15 \rho(\tau)
\end{aligned}
$$

Substitution of the expressions (24a)-(25) for $E\left[\xi^{n} \xi_{\tau}^{m}\right]$ in (5) yields the following expression for $R_{y}(\tau)$ :

$$
\begin{aligned}
R_{y}(\tau)= & \alpha_{1,1} \rho(\tau) \sigma^{2}+\left[\alpha_{3,1} \rho(\tau)+\alpha_{3,3} \rho^{3}(\tau)\right] \sigma^{4} \\
& +\left[\alpha_{5,1} \rho(\tau)+\alpha_{5,3} \rho^{3}(\tau)+\alpha_{5,5} \rho^{5}(\tau)\right] \sigma^{6} \\
& +\cdots
\end{aligned}
$$

where the first few coefficients $\alpha_{i, j}$ are evaluated in (27) and are given by

$$
\begin{aligned}
& \alpha_{1,1}=K_{1}^{2}, \\
& \alpha_{3,1}=6 K_{1} K_{3} ; \\
& \alpha_{3,3}=0, \\
& \alpha_{5,1}=\left[30 K_{1} K_{5}+9 K_{3}^{2}\right] ; \\
& \alpha_{5,3}=6 K_{3}^{2} ; \\
& \alpha_{5,5}=0 .
\end{aligned}
$$

\section{Power Spectral Density of the Amplifier Output Process}

The power spectral density $P_{y}(f)$ of the amplifier output process is obtained by taking the Fourier transform on both sides of (26) which results in

$$
\begin{aligned}
P_{y}(f) & \\
= & \alpha_{1,1} P_{\xi}(f) \sigma^{2}+\left[\alpha_{3,1} P_{\xi}(f)+\alpha_{3,3}{ }^{3} P_{\xi}(f)\right] \sigma^{4} \\
& +\left[\alpha_{5,1} P_{\xi}(f)+\alpha_{5,3}{ }^{3} P_{\xi}(f)+\alpha_{5,5}{ }^{5} P_{\xi}(f)\right] \sigma^{6} \\
& +\cdots .
\end{aligned}
$$

In $(28){ }^{k} P_{\xi}(f)$ for any positive integer $k$ denotes the $k$ times convolution of $P_{\xi}(f)$ with itself $k$ times. Thus

$$
\begin{gathered}
{ }^{2} P_{\xi}(f)=P_{\xi}(f) \otimes P_{\xi}(f) ; \\
{ }^{3} P_{\xi}(f)=P_{\xi}(f) \otimes P_{\xi}(f) \otimes P_{\xi}(f) ;
\end{gathered}
$$

where $\otimes$ denotes convolution. Direct convolution of the PSD of the input bandpass process $P_{\xi}(f)$ with itself multiple times results in spectral zones centered around frequencies that are multiples of the input signal center frequency $f_{c}$. In most applications including the NPR evaluation, only the PSD in the fundamental zone is of interest. In this case the convolution process can be significantly simplified using the following approach. The correlation function of the input bandpass process $\rho(\tau)$ is written as

$$
\rho(\tau)=\rho_{b}(\tau) \cos \left(2 \pi f_{c} \tau\right),
$$


where $\rho_{b}(\tau)$ denotes the autocorrelation function of the baseband process $\xi_{b}(t)$ corresponding to the input process $\xi(t)$ by

$$
\xi(t)=\sqrt{2} \xi_{b}(t) \cos \left(2 \pi f_{c} t\right) .
$$

From (30) the PSD of $\xi_{b}(t)$ denoted by $P_{\xi_{b}}(f)$ is related to $P_{\xi}(f)$ by

$$
P_{\xi}(f)=\frac{1}{2} P_{\xi_{b}}\left(f-f_{c}\right)+\frac{1}{2} P_{\xi_{b}}\left(f+f_{c}\right) .
$$

It is also apparent from (30) or (32) that $E\left[\xi^{2}\right]=E\left[\xi_{b}^{2}\right]$. It follows from (30) that

$$
\begin{aligned}
\rho^{3}(\tau) & =\rho_{b}^{3}(\tau) \cos ^{3}\left(2 \pi f_{c} \tau\right) \\
& =\rho_{b}^{3}(\tau)\left[0.75 \cos \left(2 \pi f_{c} \tau\right)+0.25 \cos \left(6 \pi f_{c} \tau\right)\right]
\end{aligned}
$$

Taking Fourier transform on both sides of (33) it follows that the one-sided fundamental zone component of the PSD ${ }_{s}^{3} P_{\xi}(f)$ is given by

$$
{ }_{s}^{3} P_{\xi, f z}(f)=0.75{ }^{3} P_{\xi_{b}}\left(f-f_{c}\right) .
$$

Thus except for the constant 0.75 , the one-sided fundamental zone component of the PSD ${ }_{s}^{3} P_{\xi}(f)$ is obtained by simply shifting the corresponding (two-sided) baseband PSD ${ }^{3} P_{\xi_{b}}(f)$, where

$$
\begin{gathered}
{ }^{2} P_{\xi_{b}}(f)=P_{\xi_{b}}(f) \otimes P_{\xi_{b}}(f) \\
{ }^{3} P_{\xi_{b}}(f)=P_{\xi_{b}}(f) \otimes P_{\xi_{b}}(f) \otimes P_{\xi_{b}}(f)
\end{gathered}
$$

In general the one-sided fundamental zone component of the PSD ${ }_{s}^{k} P_{\xi}(f)$ is obtained by simply shifting the corresponding (two-sided) baseband $\mathrm{PSD}^{k} P_{\xi_{b}}(f)$ by

$$
{ }_{s}^{k} P_{\xi, f z}(f)=a_{k, 1}{ }^{k} P_{\xi_{b}}\left(f-f_{c}\right) ; \quad k=1,3,5, \ldots,
$$

where $a_{k, 1}$ is the coefficient of $\cos \left(2 \pi f_{c} t\right)$ in the trigonometric expansion of $\cos ^{k}\left(2 \pi f_{c} t\right)$ in

$$
\begin{aligned}
\cos ^{k}\left(2 \pi f_{c} t\right)= & a_{k, 1} \cos \left(2 \pi f_{c} t\right)+a_{k, 3} \cos \left(6 \pi f_{c} t\right) \\
& +\cdots .
\end{aligned}
$$

These coefficients $a_{k, j}$ for any odd integer can be obtained by the following recursion [1]:

$$
\begin{aligned}
& a_{k+2,1}=0.75 a_{k, 1}+0.25 a_{k, 3} \\
& a_{k+2, j}=0.5 a_{k, j}+0.25 a_{k, j-2}
\end{aligned}
$$$$
1<j \leq k ; j: \text { odd }
$$

$$
a_{k+2, k+2}=0.25 a_{k, k}
$$

with $a_{1,1}=1$ and $a_{k, j}=0$ for $j>k$.
In summary the PSD of the amplifier output fundamental zone component of the bandpass power spectral density ${ }_{s}^{k} P_{\xi}(f)$ is obtained by first obtaining the corresponding baseband (two-sided) power spectral density $P_{\xi_{b}}(f)$ by shifting the one-sided PSD $2 P_{\xi}(f)$ to $f$ equal to 0 , convolving $P_{\xi_{b}}(f)$ with itself $k$ times, and scaling the result of convolution by the constant $a_{k, 1}$ and finally shifting the result to the input signal center frequency $f_{c}$. This process also results in a considerable simplification in the actual numerical evaluation of the result.

\section{Numerical Results}

This section presents some numerical results obtained by using the analysis presented in the paper. The analytical results have been encoded in the form of a relatively simple MATLAB program that can provide the result for any specified amplifier input-output characteristics, notch bandwidth and depth, and the output power back-off. The MATLAB program then generates the desired numerical result almost instantaneously.

The first example generates the various terms ${ }^{k} P(\xi)$ appearing in the expression for the amplifier output power spectral density $P_{y}(f)$ in (28) for various values of the integer $k$ for the case wherein the input is a band limited white noise with a specified notch in the spectral band of the input noise. Thus the result of Example 1 may be applied to any specific nonlinear amplifier. The second example considers an example amplifier input-output characteristics and evaluates the noise power ratio (NPR) as a function of the output backoff using the result of Example 1. The NPR ratio is the ratio of the maximum PSD outside and in the vicinity of the notch band and the PSD in the center of the notch band at the output of the nonlinear amplifier.

Example 1. The process $\xi(t)$ is a band limited white noise with power $\sigma_{\xi}^{2}$ equal to $1 \mathrm{~W}$ and bandwidth $B_{T}$ equal to $100 \mathrm{MHz}$ and has a notch of bandwidth $2 \mathrm{MHz}$ and $80 \mathrm{~dB}$ depth. In the example, the power level is adjusted to $1 \mathrm{~W}$ after the notch. Figure 1 shows the one-sided PSD $2 P_{\xi}(f)$ shifted to $f$ equal to 0 . The figure also plots the corresponding fundamental zone component of the bandpass power spectral density ${ }^{k} P_{\xi}(f)$ for the order $k$ equal to 3, 5, and 7. All the PSDs in the figure are the one-sided PSDs shifted to $f$ equal to 0 . Figure 2 shows the corresponding result in $\mathrm{dB}$ scale over a $4 \mathrm{MHz}$ band around the notch.

It may be observed from Figure 1 that there is only a small difference in the power spectral density inside the notch and at the border of the notch for the third-order term and a negligible difference in the higher order terms. Figure 3 shows the result of Figure 2 over a bandwidth of $200 \mathrm{MHz}$ that is two times the input noise bandwidth. Figure 2 shows the details of the PSD of various terms in the immediate vicinity of the notch.

Example 2. As an example of the application of the theory presented in this paper, the nonlinear amplifier is approximated by a soft limiter model with its input-output 


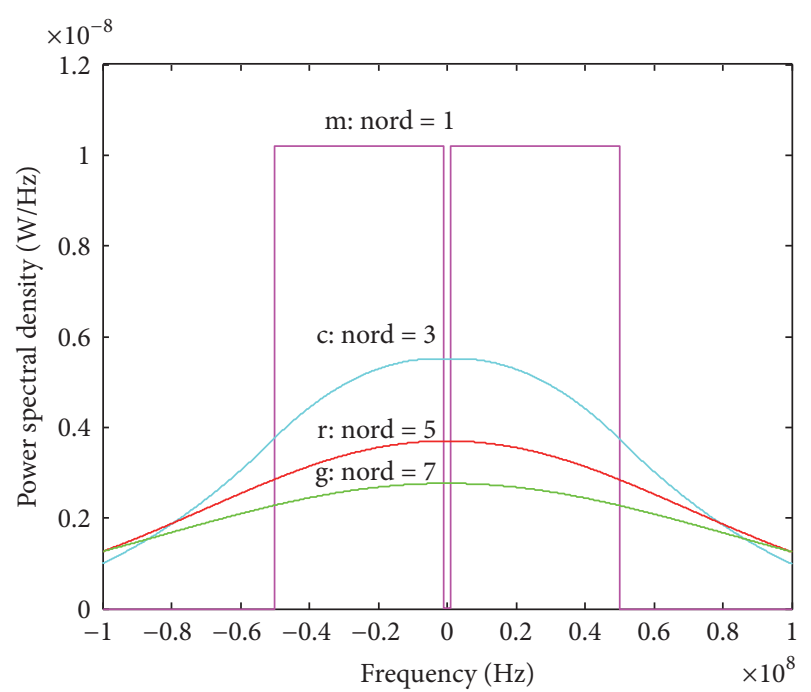

Figure 1: Power spectral density $(\mathrm{W} / \mathrm{Hz})$ of various order convolution terms $\left(B_{T}=100 \mathrm{MHz}, B_{\text {notch }}=2 \mathrm{MHz}\right.$, and $\left.\sigma_{\xi}^{2}=1 \mathrm{~W}\right)$.

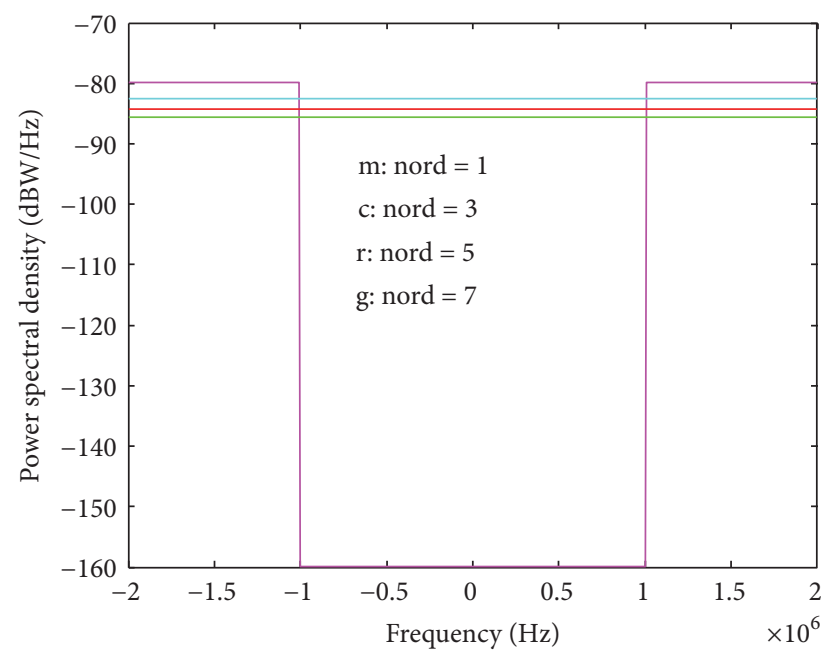

FIgURE 2: Power spectral density $(\mathrm{dBW} / \mathrm{Hz})$ of various order convolution terms $\left(B_{T}=100 \mathrm{MHz}, B_{\text {notch }}=2 \mathrm{MHz}\right.$, and $\left.\sigma_{\xi}^{2}=1 \mathrm{~W}\right)$.

characteristic described by the following nonlinear function $F(u)$ given by

$$
F(u)=\operatorname{erf}\left(\frac{u}{\sqrt{2}}\right) \equiv \sqrt{\frac{2}{\pi}} \int_{0}^{u} \exp \left(-\frac{x^{2}}{2}\right) d x .
$$

Figure 4 plots the nonlinearity $F(u)$ depicting good linearity over the input range of $(-1,1)$. The function $F(u)$ may be approximated by a polynomial of sufficiently high order such that the approximation error is negligible over the specified range of $u$. When the input to the amplifier is a sine wave of constant amplitude $A$, the amplifier output contains the fundamental zone and various harmonic zones. The fundamental zone output is just a sine wave of amplitude

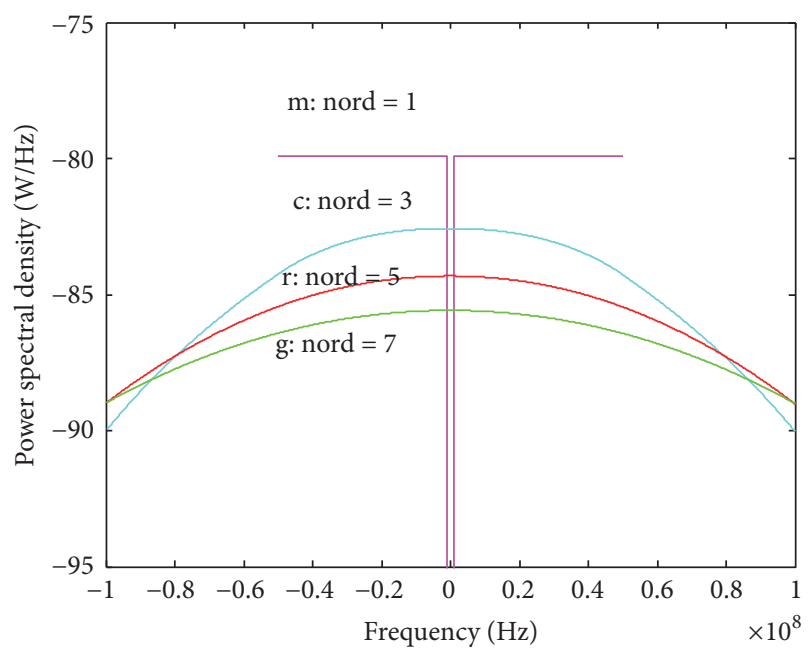

FIgure 3: Power spectral density $(\mathrm{dBW} / \mathrm{Hz})$ of various order convolution terms over a bandwidth of $200 \mathrm{MHz}\left(B_{T}=100 \mathrm{MHz}\right.$, $B_{\text {notch }}=2 \mathrm{MHz}$, and $\left.\sigma_{\xi}^{2}=1 \mathrm{~W}\right)$.

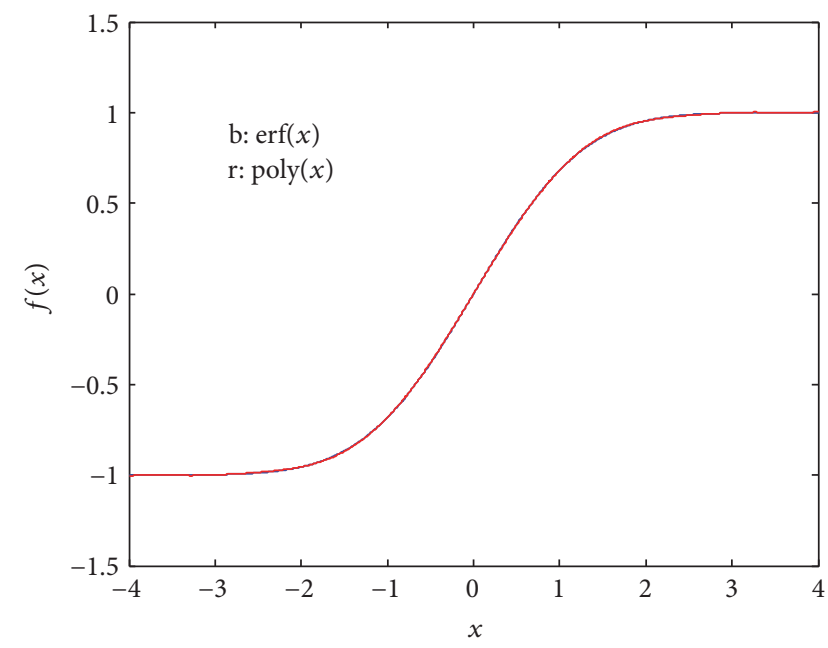

FIGURE 4: Soft limiter input-output characteristics.

$g(A)$, which is given by the Chebyshev transform [16] of $F(u)$ and for the specific case of (39) is given by

$$
g(A)=\sqrt{\frac{2}{\pi}} A \exp \left(-\frac{A^{2}}{4}\right)\left[I_{0}\left(\frac{A^{2}}{4}\right)+I_{1}\left(\frac{A^{2}}{4}\right)\right] .
$$

In (40) $I_{0}$ and $I_{1}$ are the modified Bessel functions of zero and first order, respectively. Figure 5 plots the function $g(A)$ versus $A$ for the soft limiter. In addition, Figure 6 plots the input signal power level $P_{i}$ equal to $A^{2} / 2$ versus the amplifier output signal power equal to $(g(A))^{2} / 2$ in dB scale.

The amplifier characteristics $F(u)$ are approximated by a polynomial of sufficiently high degree over the range of input signal which is taken equal to $3 \sigma$, where $\sigma^{2}$ is the variance or power $P_{i}$ of the zero mean Gaussian input noise process $u(t)$. Figure 7 shows the power spectral density of the amplifier output $y(t)$ computed from (28) with $P_{i}=0 \mathrm{dBW}$. The coefficients $K_{1}, K_{3}, \ldots$ are obtained by a degree 9 polynomial 


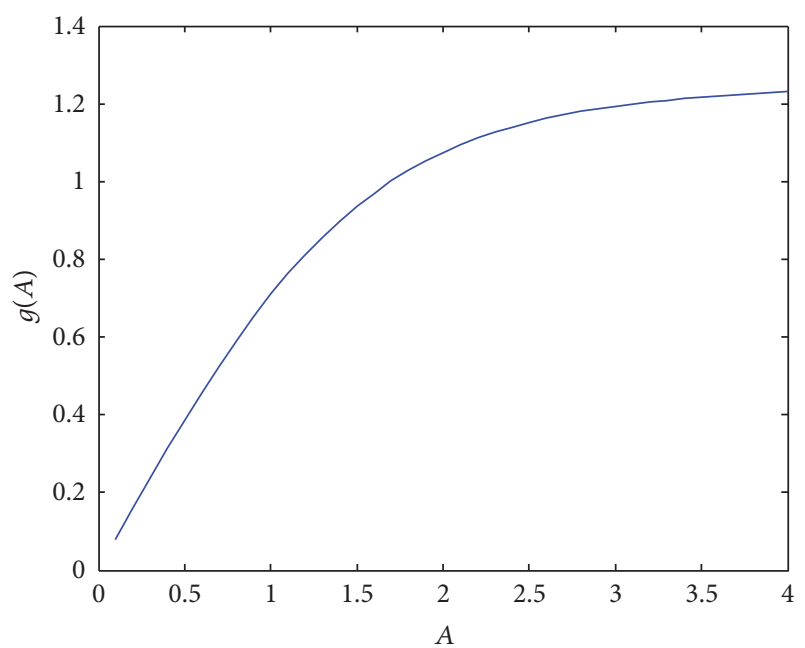

Figure 5: Plot of the Chebyshev transform $g(A)$.

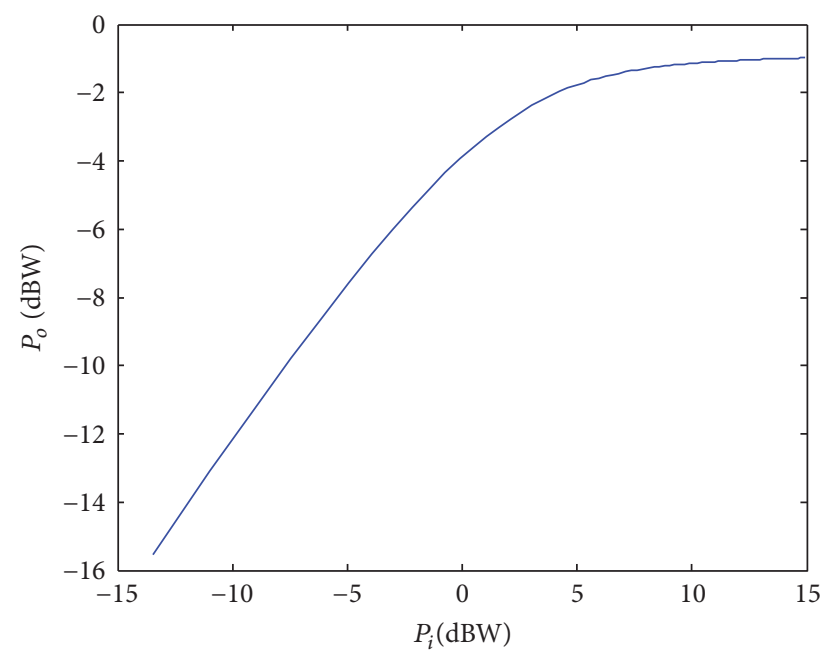

Figure 6: Amplifier output versus input power level.

approximation of $F(u)$ over the interval $(-3,3)$ and are equal to $\left[\begin{array}{lllll}0.7962 & -0.1284 & 0.0165 & -0.0013 & 4.12 \times 10^{-5}\end{array}\right]$. The RMS approximation error in the polynomial approximation is equal to $3.38 \times 10^{-4}$ thus achieving very good curve fit.

Figures 8 and 9 plot the corresponding results for the case of $P_{i}$ equal to $-3 \mathrm{dBW}$ and $-4 \mathrm{dBW}$, respectively. The amplifier coefficients $K_{1}, K_{3}, \ldots$ for the two cases are equal to $\left[\begin{array}{lllll}0.7978 & -0.1324 & 0.0191 & -0.0019 & 9.26 \times 10^{-5}\end{array}\right]$ and $\left[\begin{array}{lllll}0.7978 & -0.1327 & 0.0195 & -0.0020 & 1.11 \times 10^{-4}\end{array}\right]$, respectively. The corresponding RMS approximation error is equal to $3.38 \times 10^{-4}$ and $1.76 \times 10^{-5}$, respectively, for the two cases. Figure 10 plots the corresponding result for the case of notch bandwidth equal to $8 \mathrm{MHz}$.

The NPR values obtained for the three cases shown in Figures 7-9 are equal to $6.09,15.38$, and $18.49 \mathrm{~dB}$, respectively. Figure 10 shows the output noise power spectral density ratio when the notch bandwidth is increased to $8 \mathrm{MHz}$ for the case of $P_{i}=-3 \mathrm{dBW}$ depicting an NPR of $15.96 \mathrm{~dB}$. Comparing the result of Figure 8 for the case of $B_{\text {notch }}=2 \mathrm{MHz}$ shows that

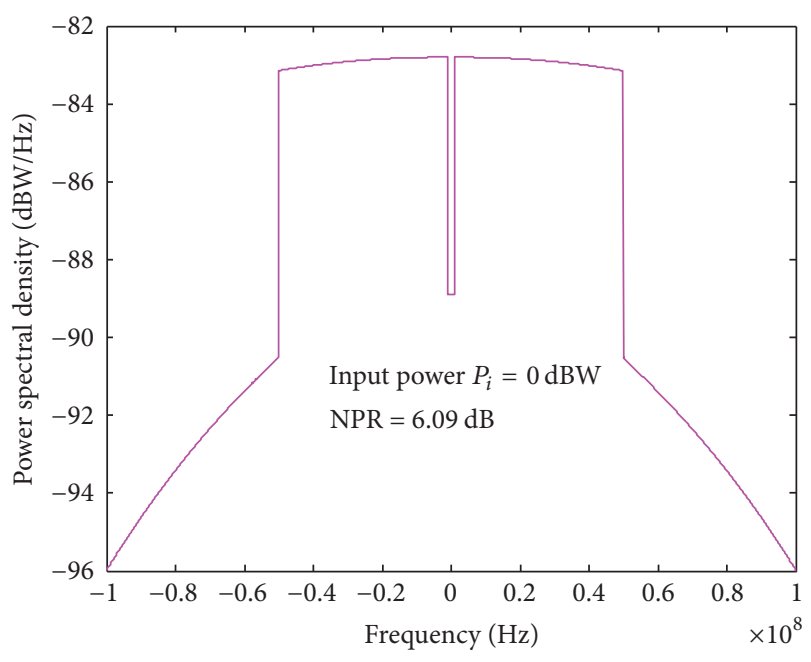

FIgURe 7: Amplifier output noise power spectral density with $P_{i}=$ $0 \mathrm{dBW}, B_{\text {notch }}=2 \mathrm{MHz}$, and notch depth $=80 \mathrm{~dB}$.

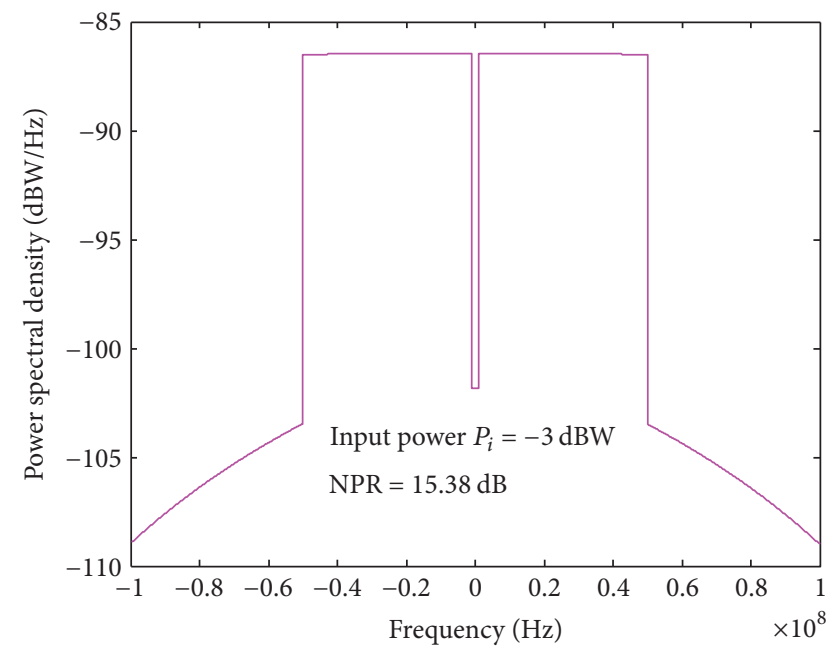

FIGURE 8: Amplifier output noise power spectral density with $P_{i}=$ $-3 \mathrm{dBW}, B_{\text {notch }}=2 \mathrm{MHz}$, and notch depth $=80 \mathrm{~dB}$.

the difference in the NPR values for the two cases is about $0.58 \mathrm{~dB}$.

Figure 11 plots the NPR versus the input power level for the three cases of $B_{\text {notch }}$ equal to $1 \mathrm{MHz}, 2 \mathrm{MHz}$, and $8 \mathrm{MHz}$, respectively. There is no significant difference in the NPR between the two cases of $1 \mathrm{MHz}$ and $2 \mathrm{MHz}$ notch bandwidth while for the case of $8 \mathrm{MHz}$ bandwidth the NPR is about $1 \mathrm{~dB}$ higher. As may be inferred from Figure 11, the plots of NPR versus $P_{i}$ have a slope of about $-3.1 \mathrm{~dB} / \mathrm{dB}$ for power levels higher than $-5 \mathrm{dBW}$ and a slope of about $-2.16 \mathrm{~dB} / \mathrm{dB}$ for lower values of $P_{i}$.

Figure 12 plots the NPR versus the output power backoff $\mathrm{BO}$ for the three cases of $B_{\text {notch }}$ equal to $1 \mathrm{MHz}, 2 \mathrm{MHz}$, and $8 \mathrm{MHz}$, respectively. The output power back-off $\mathrm{BO}$ is defined as the amplifier output signal power level relative to the fundamental zone power of a hard limiter with sine wave input which is equal to $8 / \pi^{2}=-0.9 \mathrm{~dB}$. Figure 12 also 


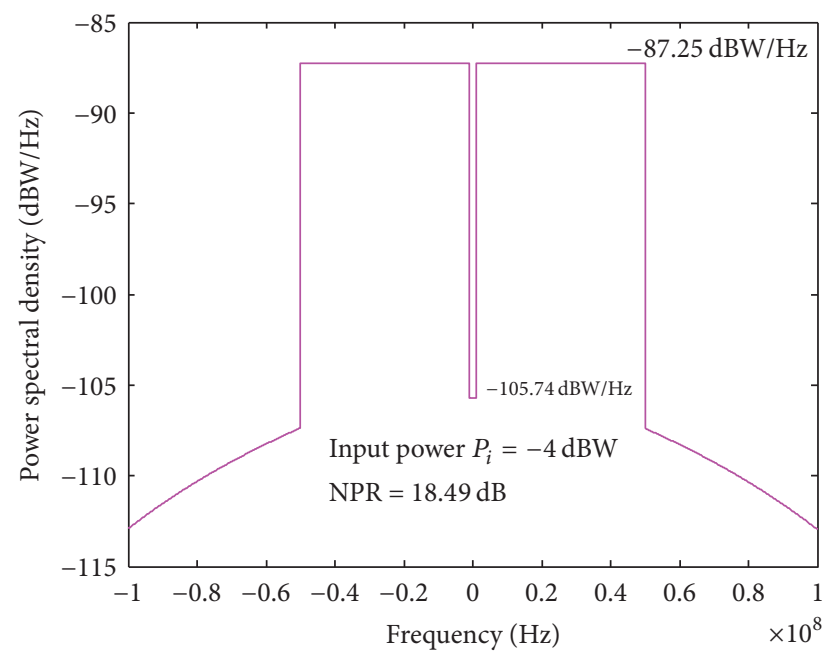

Figure 9: Amplifier output noise power spectral density with $P_{i}=$ $-4 \mathrm{dBW}, B_{\text {notch }}=2 \mathrm{MHz}$, and notch depth $=80 \mathrm{~dB}$.

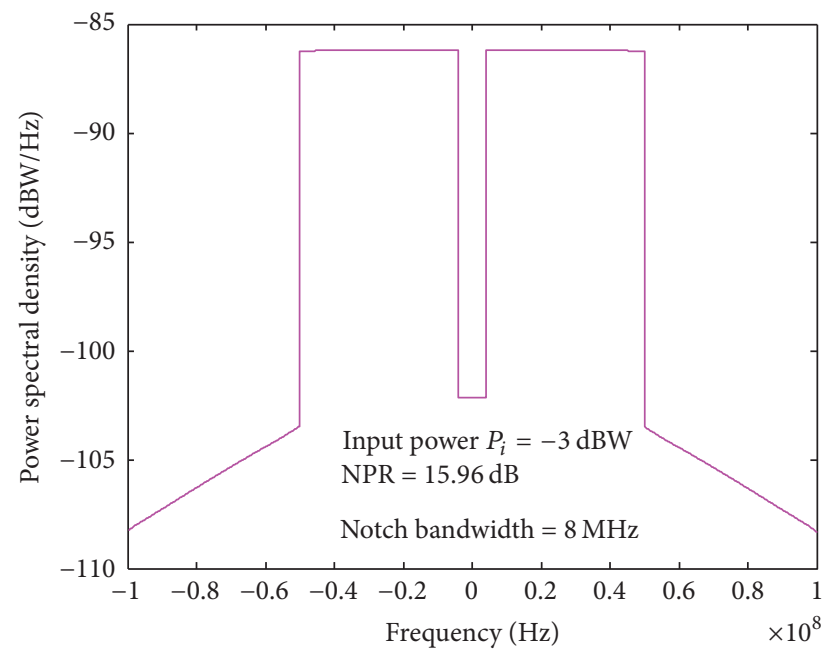

FIgURE 10: Amplifier output noise power spectral density with $P_{i}=$ $-3 \mathrm{dBW}, B_{\text {notch }}=8 \mathrm{MHz}$, and notch depth $=80 \mathrm{~dB}$.

plots the result for the amplifier output signal to distortion power ratio for the case of $M$ equal to 256 CDMA signals with QPSK modulation obtained from the analysis in [1]. The result for CDMA is close to that of the NPR result of the paper providing a cross-validation of the result as for relatively higher value of $M$ the CDMA signal may be approximated by a Gaussian distribution.

\section{Conclusions}

This paper has presented a theoretical analysis of the amplifier output noise power spectral density result in a closed form from which the NPR is evaluated by a simple subtraction. The method can be applied to any amplifier with known inputoutput characteristics. The paper has presented numerical results on the NPR as a function of the noise bandwidth, depth level of the notch, and the output power back-off

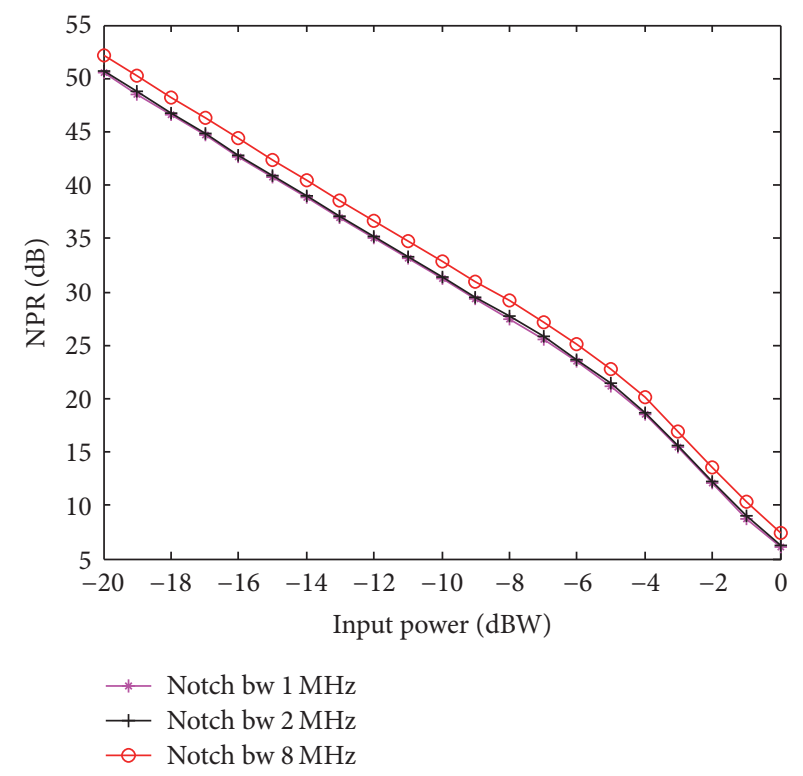

FIGURE 11: Plot of NPR versus the input power level.

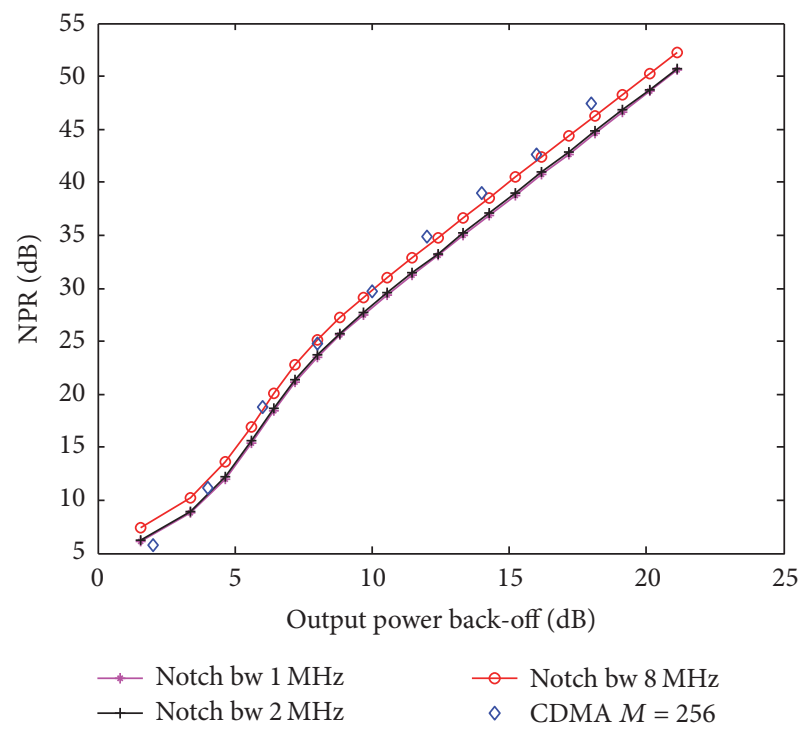

Figure 12: Plot of NPR versus the output power back-off BO.

obtained from the analysis presented in the paper. The analytical results of the paper have been encoded in the form of a MATLAB program that can provide the result for any specified amplifier input-output characteristics, notch bandwidth and depth, and the output power back-off. The MATLAB program generates the desired numerical result almost instantaneously compared to extensive efforts and time required for obtaining experimental and simulation results for any power amplifier.

\section{Competing Interests}

The author declares that there is no conflict of interests regarding the publication of this paper. 


\section{References}

[1] R. Kumar, "A high-order analysis of the distortion effects of nonlinear amplifiers on QPSK-modulated CDMA signals," in Proceedings of the AIAA International Communication Satellite Systems Conference, pp. 1-14, San Diego, Calif, USA, June 2008.

[2] R. Kumar, "A higher-order analysis of the distortion effects of nonlinear amplifiers on CDMA signals," in Proceedings of the IEEE Aerospace Conference, pp. 1-10, Big Sky, Mont, USA, March 2008.

[3] R. Kumar, "An analysis of the distortion effects of nonlinear amplifiers on CDMA signals," in Proceedings of the IEEE Aerospace Conference, pp. 1-11, Big Sky, Mont, USA, March 2007.

[4] A. Conti, D. Dardari, and V. Tralli, "On the analysis of single and multiple carrier WCDMA systems with polynomial nonlinearities," in Proceedings of the Joint Conference of the 4th International Conference on Information, Communications and Signal Processing, and 4th Pacific Rim Conference on Multimedia, vol. 1, pp. 369-375, 2003.

[5] A. Conti, D. Dardari, and V. Tralli, "An analytical framework for CDMA systems with a nonlinear amplifier and AWGN," IEEE Transactions on Communications, vol. 50, no. 7, pp. 1110-1120, 2002.

[6] A. Silberger, "The effect of nonlinear amplifiers on orthogonalcdma link and measures of performance, using a simplified amplifier model," in Proceedings of 6th IEEE International Conference on Universal Personal Communications (ICUPC '97), pp. 521-524, IEEE, Chicago, Ill, USA, October 1997.

[7] D. Dardari, "Spectral properties and signal-to-distortion ratio of arbitrary spectrum gaussian signals in the presence of nonlinearities," in Proceedings of the IEEE Global Telecommunications Conference (GLOBECOM '01), vol. 2, pp. 1147-1151, San Antonio, Tex, USA, November 2001.

[8] N. Y. Ermolova, "Spectral analysis of nonlinear amplifier based on the complex gain Taylor series expansion," IEEE Communications Letters, vol. 5, no. 12, pp. 465-467, 2001.

[9] D. Dardari, V. Tralli, and A. Vaccari, "A theoretical characterization of nonlinear distortion effects in OFDM systems," IEEE Transactions on Communications, vol. 48, no. 10, pp. 1755-1764, 2000.

[10] E. Costa, M. Midrio, and S. Pupolin, "Impact of amplifier nonlinearities on OFDM transmission system performance," IEEE Communications Letters, vol. 3, no. 2, pp. 37-39, 1999.

[11] R. De Gaudenzi and A. Silberger, "Payload non linearity effects on the globalstar forward link," in Proceedings of the IEEE International Conference on Universal Personal Communications (ICUPC '97), pp. 311-315, Chicago, Ill, USA, October 1997.

[12] D. Boudreau, G. Caire, G. E. Corazza et al., "Wide-band CDMA for the UMTS/IMT-2000 satellite component," IEEE Transactions on Vehicular Technology, vol. 51, no. 2, pp. 306-331, 2002.

[13] O. Shimbo, Transmission Analysis in Communication Systems, vol. 2, Computer Science Press, Rockville, Md, USA, 1988.

[14] M. C. Jeruchim, P. B. Balaban, and K. S. Shanmugan, Simulation of Communication Systems, Plenum Press, 2000.

[15] S. C. Cripps, Advanced Techniques in RF Power Amplifier Design, Artech House, 2002.

[16] N. M. Blachman, "Detectors, bandpass nonlinearities, and their optimization: inversion of the chebyshev transform," IEEE Transactions on Information Theory, vol. IT-17, no. 4, pp. 398404, 1971.
[17] J. J. Spilker, Digital Communications by Satellite, Prentice Hall, 1977.

[18] N. Wax, Ed., Selected Papers on Noise and Stochastic Processes, Dover Publications, New York, NY, USA, 1954.

[19] S. O. Rice, "Mathematical analysis of random noise," The Bell System Technical Journal, vol. 23, pp. 282-332, 1944. 


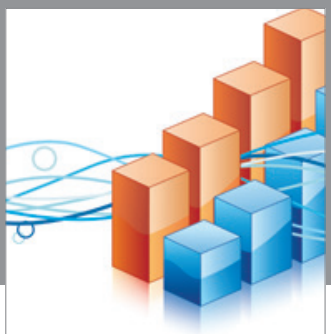

Advances in

Operations Research

vatem alat4

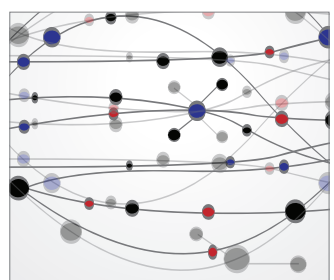

\section{The Scientific} World Journal
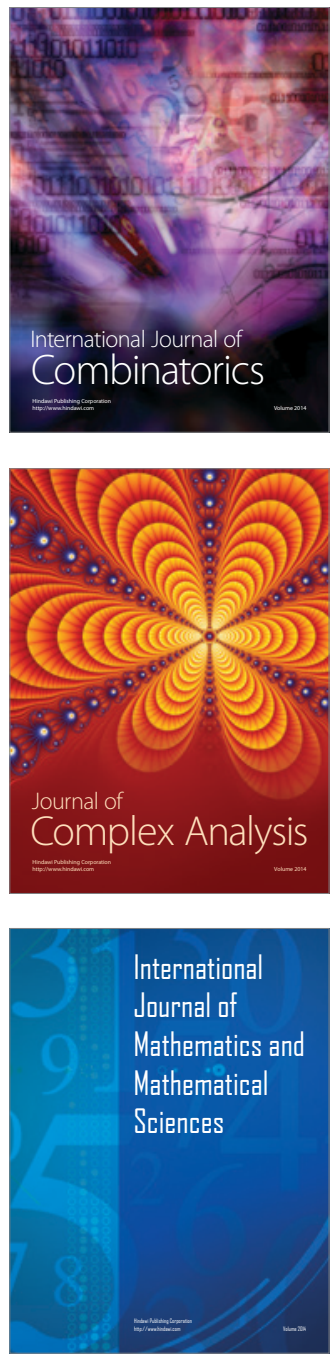
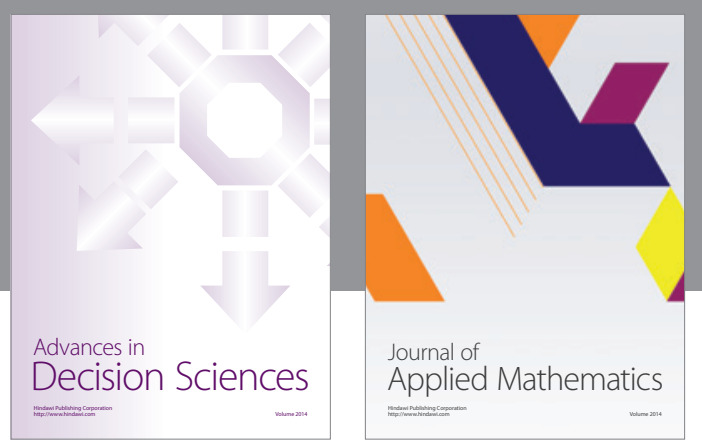

Algebra

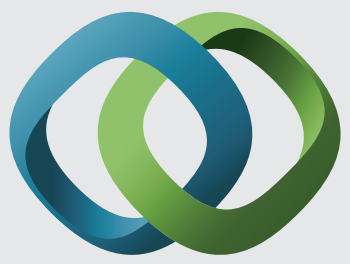

\section{Hindawi}

Submit your manuscripts at

http://www.hindawi.com
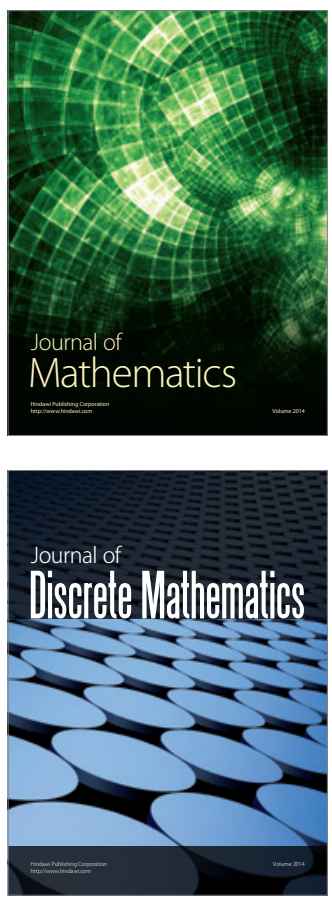

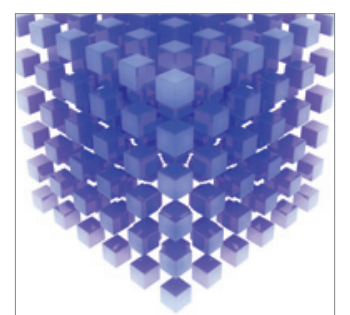

Mathematical Problems in Engineering
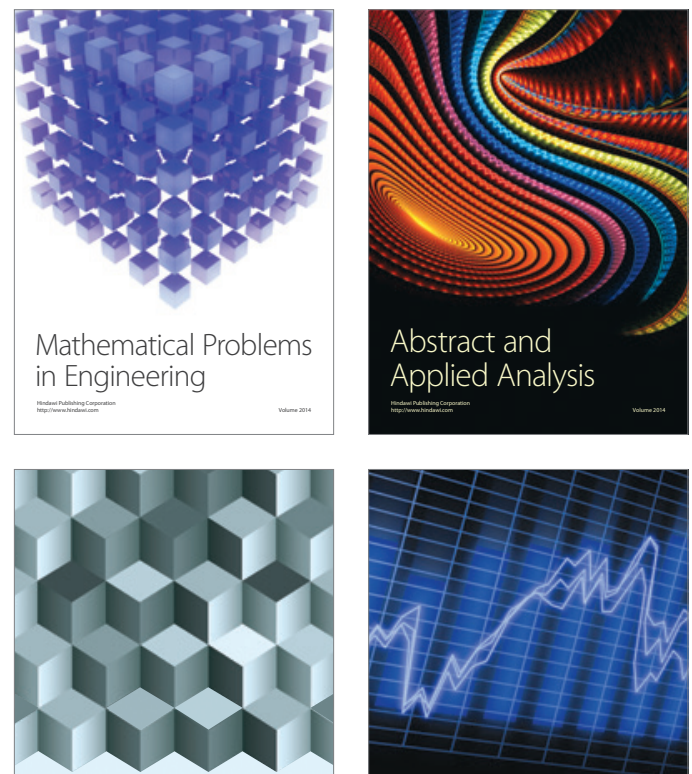

Journal of

Function Spaces

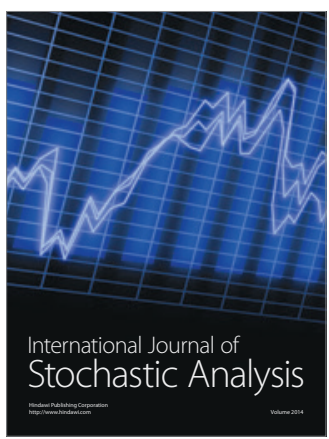

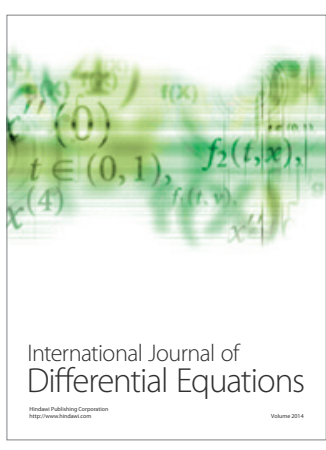
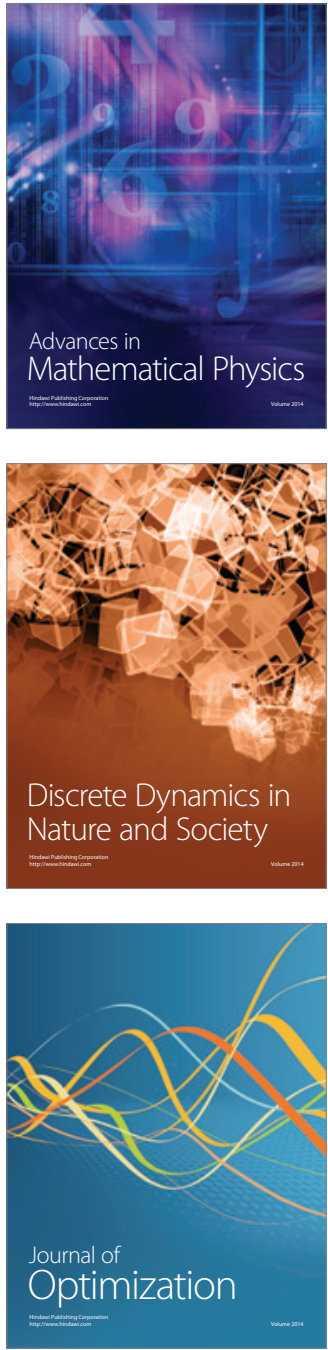